\title{
Pneumoscrotum associated with nontherapeutic lower gastrointestinal endoscopy
}

A 66-year-old man underwent flexible rectosigmoidoscopy due to anemia and rectal bleeding. The rectosigmoidoscopy showed blood oozing from third-degree hemorrhoids ( Fig. 1 a). During the procedure the patient noticed swelling of his scrotum ( $\bullet$ Fig. 1 b); he then developed abdominal pain. A computed axial tomography scan confirmed subcutaneous emphysema and the presence of pneumoperitoneum, with air in the scrotum ( $\bullet$ Fig. 2 a), around the rectum ( $\bullet$ Fig. $\mathbf{2 b}$ ), in the retroperitoneum, and the perinephric ( $\bullet$ Fig. 2 c) as well as the paraesophageal ( $\bullet$ Fig. $2 \mathbf{d}$ ) spaces. Physical examination under general anesthesia revealed perforation of the rectum into the subcutaneous tissue adjacent to the hemorrhoids. After consultation with the surgeons, the patient was kept nil per os overnight. Antibiotics were administered (ciprofloxacin $200 \mathrm{mg}$ i.v. bid, metronidazole $500 \mathrm{mg}$ i.v. tid), and the pneumoscrotum resolved within 3 days. The patient's white blood cell count remained stable. After 3 days, the patient was started on a full liquid diet and then advanced to a regular diet. He was discharged uneventfully after 4 days in hospital.

Subcutaneous or retroperitoneal air that dissects into the dartos lining of the scrotal wall or movement of air from the intraperitoneal space into the scrotum may result in pneumoscrotum. Local gas production is another mechanism that suggests anaerobic infection, requiring urgent surgical interventions [1]. In the literature there are only seven cases of pneumoscrotum following lower gastrointestinal endoscopy [2-8]. Four of these patients had undergone polypectomy [2-5], one had undergone endoscopic mucosal resection for a tumor [6], and one with ulcerative colitis had undergone biopsy [7]. To the best of our knowledge, our case is only the second one reported in the literature involv-

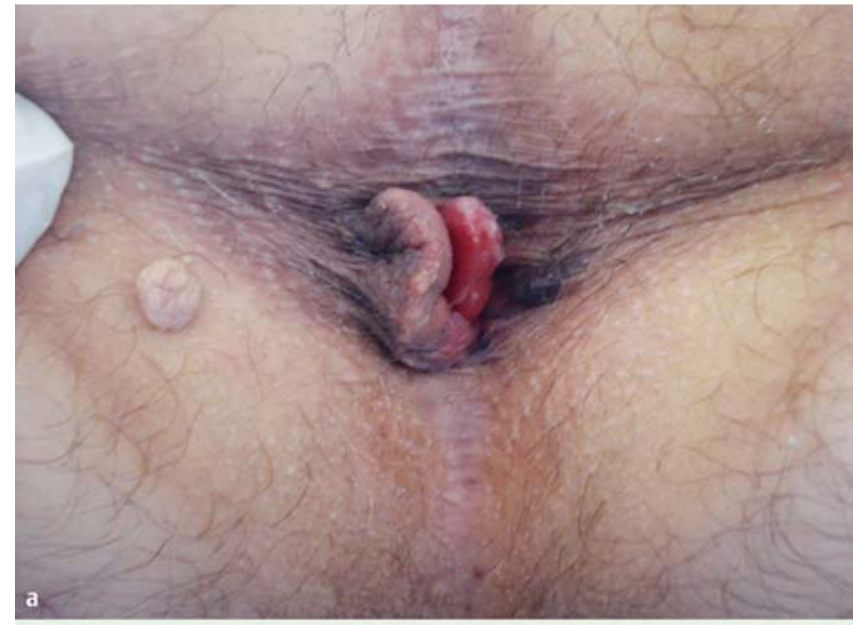

Fig. 1 Physical examination following rectosigmoidoscopy. a External hemorrhoids were visible on perianal inspection in the kneeelbow position.

b Edematous scrotum.
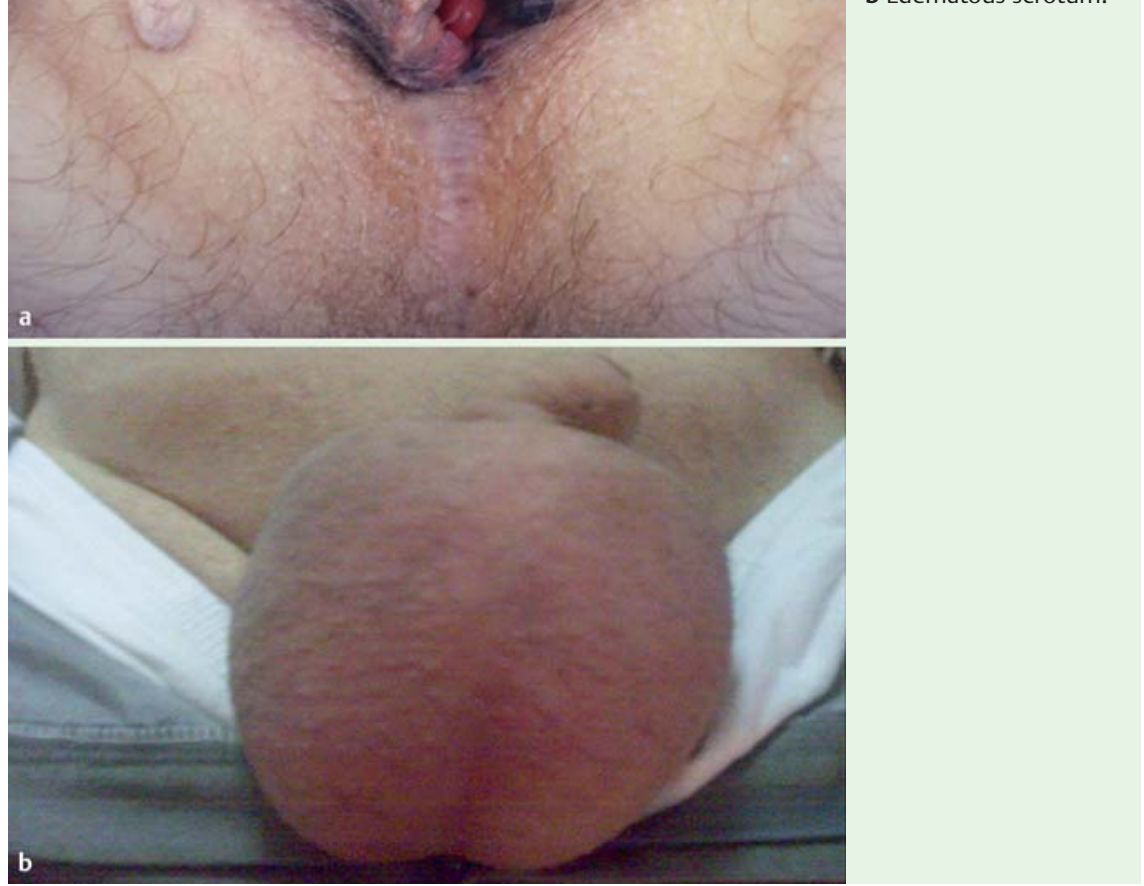

ing the development of pneumoscrotum following a pure diagnostic lower gastrointestinal endoscopy [8]. None of the patients with this complication required a surgical repair procedure, and complete resolution was observed after close clinical observation, with judicious use of antibiotics and serial monitoring of the patients. Our case serves as an important reminder to gastroenterologists that pneumoscrotum could present as a sign of colonic perforation even in patients with diagnostic lower gastrointestinal endoscopy.

Endoscopy_UCTN_Code_CPL_1AJ_2AB
M. Akdogan ${ }^{1}$, I. K. Onal ${ }^{1}$, M. Kurt ${ }^{1}$, Z. M. Yalinkilic ${ }^{1}$, A. Sayilir ${ }^{1}$, K. Karaman ${ }^{2}$, B. Celep ${ }^{2}$, R. S. Okten ${ }^{3}$

1 Department of Gastroenterology, Turkiye Yuksek Ihtisas Teaching and Research Hospital, Ankara, Turkey

2 Department of Gastrointestinal Surgery, Turkiye Yuksek Ihtisas Teaching and Research Hospital, Ankara, Turkey

3 Department of Radiology, Turkiye Yuksek Ihtisas Teaching and Research Hospital, Ankara, Turkey 

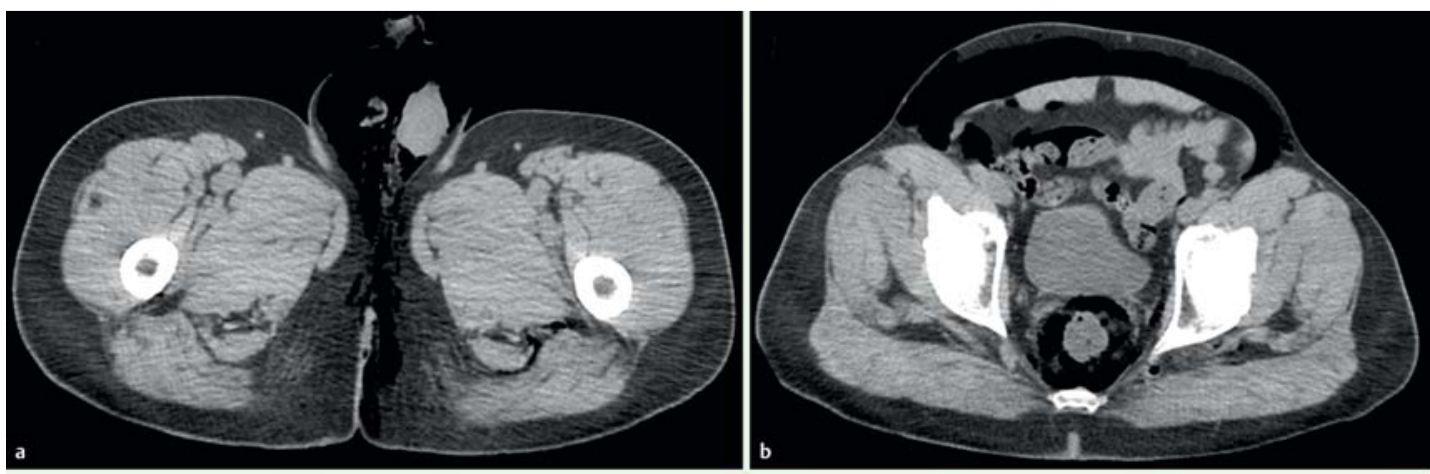

Fig. 2 Computed tomography scans of the thorax and abdomen demonstrated: a pneumoscrotum; b free air surrounding the rectal wall, c in the perinephric area on both sides, d and in the paraesophageal space.
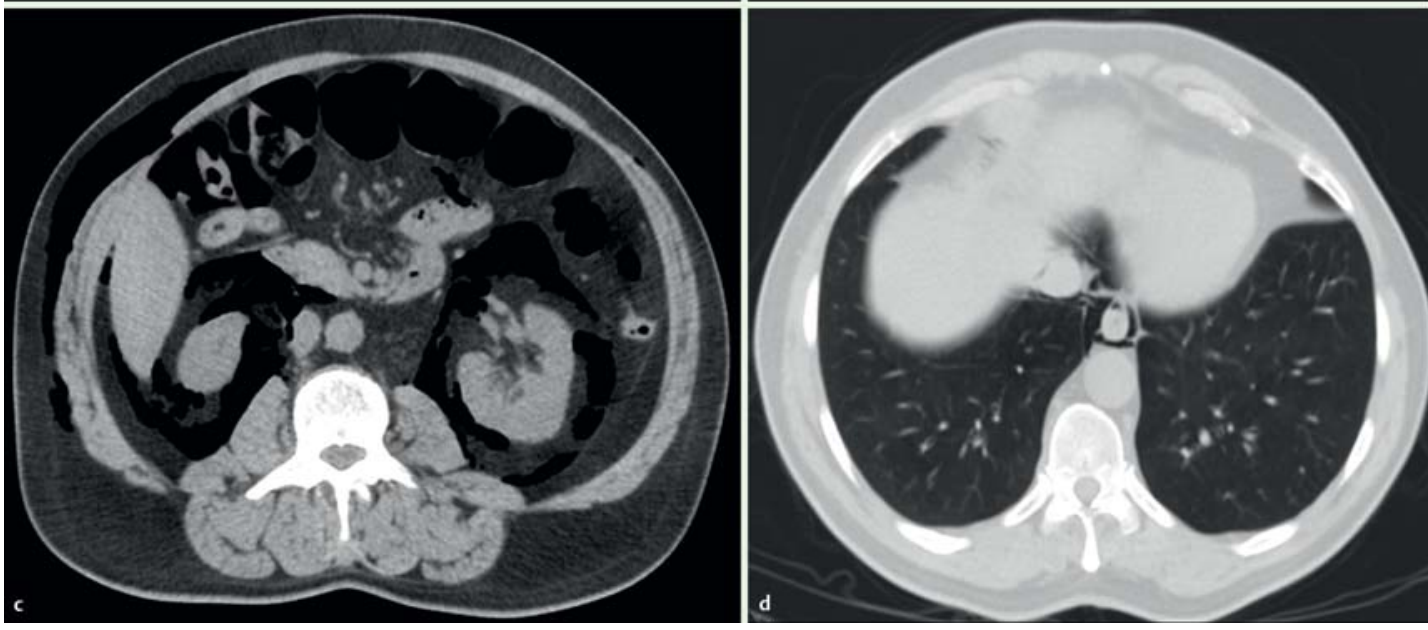

\section{References}

1 Watson HS, Klugo RG, Coffield KS. Pneumoscrotum: report of two cases and review of mechanisms of its development. Urology 1992; 40: 517-521

2 Singh S, Thakur M. Pneumoscrotum after colonoscopy. Can J Gastroenterol 2008; 22: 411-413

3 Humphreys F, Hewetson KA, Dellipiani AW. Massive subcutaneous emphysema following colonoscopy. Endoscopy 1984; 16: $160-161$

4 Carlsen CU, Andreassen KH. Benign pneumoperitoneum and scrotal emphysema after colonoscopy. Ugeskr Laeger 1992; 154: $1785-1786$
5 Graepler R, Lauer UM, Horger MS et al. Pneumoscrotum after colonoscopic polypectomy. Endoscopy 2007; 39: E90

6 Fu KI, Sano Y, Kato S et al. Pneumoscrotum: a rare manifestation of perforation associated with therapeutic colonoscopy. World J Gastroenterol 2005; 11: 5061 - 5063

7 Goerg KJ, Duber C. Retroperitoneal, mediastinal and subcutaneous emphysema with pneumothorax after colonoscopy. Dtsch Med Wochenschr 1996; 121: 693-696

8 Fishman EK, Goldman SM. Pneumoscrotum after colonoscopy. Urology 1981; 18: 171 172
Bibliography

DOI 10.1055/s-0029-1215410

Endoscopy 2010; 42: E7 -E8

(c) Georg Thieme Verlag KG Stuttgart · New York . ISSN 0013-726X

\section{Corresponding author}

\section{Kurt, MD}

Department of Gastroenterology

Turkiye Yuksek Ihtisas Teaching and Research Hospital

Kızılay Sk. No:2, 06100

Sihhiye

Ankara

Turkey

Fax: +90-312-3124120

dr.mevlutkurt@gmail.com 